\title{
The VVDS type-1 AGN sample: the faint end of the luminosity function
}

\author{
A. Bongiorno ${ }^{1}$, G. Zamorani ${ }^{2}$, I. Gavignaud ${ }^{3}$, B. Marano ${ }^{1}$, S. Paltani ${ }^{4,5}$, G. Mathez ${ }^{6}$, P. Møller ${ }^{21}$, J. P. Picat ${ }^{6}$, \\ M. Cirasuolo ${ }^{7}$, F. Lamareille ${ }^{2,6}$, D. Bottini ${ }^{8}$, B. Garilli ${ }^{8}$, V. Le Brun ${ }^{9}$, O. Le Fèvre ${ }^{9}$, D. Maccagni ${ }^{8}$, R. Scaramella ${ }^{10,11}$, \\ M. Scodeggio ${ }^{8}$, L. Tresse ${ }^{9}$, G. Vettolani ${ }^{10}$, A. Zanichelli ${ }^{10}$, C. Adami ${ }^{9}$, S. Arnouts ${ }^{9}$, S. Bardelli ${ }^{2}$, M. Bolzonella ${ }^{2}$,

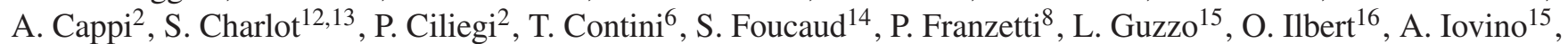 \\ H. J. McCracken ${ }^{13,17}$, C. Marinoni ${ }^{18}$, A. Mazure ${ }^{9}$, B. Meneux ${ }^{8,15}$, R. Merighi ${ }^{2}$, R. Pello ${ }^{6}$, A. Pollo ${ }^{9}, 19$, L. Pozzetti ${ }^{2}$, \\ M. Radovich ${ }^{20}$, E. Zucca ${ }^{2}$, E. Hatziminaoglou ${ }^{21}$, M. Polletta ${ }^{22}$, M. Bondi ${ }^{10}$, J. Brinchmann ${ }^{23}$, O. Cucciati ${ }^{15,24}$, \\ S. de la Torre ${ }^{9}$, L. Gregorini2 ${ }^{25}$, Y. Mellier ${ }^{13,17}$, P. Merluzzi ${ }^{20}$, S. Temporin ${ }^{15}$, \\ D. Vergani ${ }^{8}$, and C. J. Walcher ${ }^{9}$ \\ (Affiliations can be found after the references)
}

Received 4 April 2007 / Accepted 26 June 2007

\begin{abstract}
In a previous paper (Gavignaud et al. 2006, A\&A, 457, 79), we presented the type-1 Active Galactic Nuclei (AGN) sample obtained from the first epoch data of the VIMOS-VLT Deep Survey (VVDS). The sample consists of 130 faint, broad-line AGN with redshift up to $z=5$ and $17.5<I_{\mathrm{AB}}<24.0$, selected on the basis of their spectra. The sample is thus free of the morphological and color selection biases, that lead to significant incompleteness in the optical surveys of faint AGN.

In this paper we present the measurement of the Optical Luminosity Function up to $z=3.6$ derived from this sample, we compare our results with previous results from brighter samples both at low and at high redshift and finally, through the estimate of the bolometric luminosity function, we compare them also with the results from X-ray and mid-IR selected samples.

Our data, more than one magnitude fainter than previous optical surveys, allow us to constrain the faint part of the luminosity function up to high redshift. A comparison of our data with the $2 \mathrm{dF}$ sample at low redshift $(1<z<2.1)$ shows that the VVDS data can not be well fitted with the PLE models derived by previous samples. Qualitatively, this appears to be due to the fact that our data suggest the presence of an excess of faint objects at low redshift $(1.0<z<1.5)$ with respect to these models.

By combining our faint VVDS sample with the large sample of bright AGN extracted from the SDSS DR3 (Richards et al. 2006b, AJ, 131, 2766) and testing a number of different evolutionary models, we find that the model which better represents the combined luminosity functions, over a wide range of redshift and luminosity, is a luminosity dependent density evolution (LDDE) model, similar to those derived from the major Xsurveys. Such a parameterization allows the redshift of the AGN space density peak to change as a function of luminosity and explains the excess of faint AGN that we find at $1.0<z<1.5$. On the basis of this model we find, for the first time from the analysis of optically selected samples, that the peak of the AGN space density shifts significantly towards lower redshift going to lower luminosity objects. The position of this peak moves from $z \sim 2.0$ for $M_{B}<-26.0$ to $z \sim 0.65$ for $-22<M_{B}<-20$. This result, already found in a number of X-ray selected samples of AGN, is consistent with a scenario of "AGN cosmic downsizing", in which the density of more luminous AGN, possibly associated to more massive black holes, peaks earlier in the history of the Universe (i.e. at higher redshift), than that of low luminosity ones, which reaches its maximum later (i.e. at lower redshift).
\end{abstract}

Key words. surveys - galaxies: high-redshift - galaxies: quasars: general - galaxies: luminosity function, mass function

\section{Introduction}

Active galactic nuclei (AGN) are relatively rare objects that exhibit some of the most extreme physical conditions and activity known in the universe. A useful way to statistically describe AGN activity along cosmic time is through the study of their luminosity function (LF), whose shape, normalization and evolution can be used to derive constraints on models of cosmological evolution of super-massive black holes (SMBH). At $z \lesssim 2.5$, the luminosity function of optically selected type-1 AGN has been well studied for many years (Boyle et al. 1988; Hewett et al. 1991; Pei 1995; Boyle et al. 2000; Croom et al. 2004). It is usually described as a double power-law, defined by the characteristic luminosity $L^{*}$ and characteristic density $\Phi^{*}$, whose evolution with redshift allows to distinguish between simple evolutionary models such as pure luminosity evolution (PLE) and pure density evolution (PDE). Although the PLE and PDE models should be mainly considered as mathematical descriptions of the evolution of the luminosity function, two different physical interpretations can be associated to them: either a small fraction of bright galaxies harbor AGN and the luminosities of these sources change systematically with time ("luminosity evolution"), or all bright galaxies harbor AGN, but at any given time most of them are in "inactive" states. In the latter case, the fraction of galaxies with AGN in an "active" state changes with time ("density evolution"). Up to now, the PLE model is the preferred description for the evolution of optically selected QSOs, at least at low redshift $(z<2)$.

Works on high redshift type-1 AGN samples (Warren et al. 1994; Kennefick et al. 1995; Schmidt et al. 1995; Fan et al. 2001; Wolf et al. 2003; Hunt et al. 2004) have shown that the 
number density of QSOs declines rapidly from $z \sim 3$ to $z \sim 5$. Since the size of complete and well studied samples of QSOs at high redshift is still relatively small, the rate of this decline and the shape of the high-redshift luminosity function is not yet as well constrained as at low redshift. For example, Fan et al. (2001), studying a sample of 39 luminous high-redshift QSOs at $3.6<z<5.0$, selected from the commissioning data of the Sloan Digital Sky Survey (SDSS), found that the slope of the bright end of the QSO luminosity function evolves with redshift, becoming flatter at high redshift, and that the QSO evolution from $z=2$ to $z=5$ cannot be described as a pure luminosity evolution. A similar result on the flattening at high redshift of the slope of the luminosity function for luminous QSOs has been recently obtained by Richards et al. (2006b) from the analysis of a much larger sample of SDSS QSOs (but see Fontanot et al. (2007) for different conclusions drawn on the basis of combined analysis of GOODS and SDSS QSOs).

At the same time, a growing number of observations at different redshifts, in radio, optical and soft and hard X-ray bands, are suggesting that the faint end slope also evolves, becoming flatter at high redshift (Page et al. 1997; Miyaji et al. 2000, 2001; La Franca et al. 2002; Cowie et al. 2003; Ueda et al. 2003; Fiore et al. 2003; Hunt et al. 2004; Cirasuolo et al. 2005; Hasinger et al. 2005). This evolution, now dubbed as "AGN cosmic downsizing" is described either as a direct evolution in the faint-end slope, or as a luminosity-dependent density evolution (LDDE), and it has been the subject of many speculations, since it implies that the space density of low-luminosity AGN peaks at lower redshift than that of bright ones.

It has been observed that, in addition to the well known local scale relations between the SMBH masses and the properties of their host galaxies (Kormendy \& Richstone 1995; Magorrian et al. 1998; Ferrarese \& Merritt 2000), the galaxy spheroid population follows a similar pattern of "cosmic downsizing" (Cimatti et al. 2006). Various models have been proposed to explain this common evolutionary trend in AGN and spheroid galaxies. The majority of them propose that the feedback from the black hole growth plays a key role in determining the SMBH-host galaxy relations (Silk \& Rees 1998; Di Matteo et al. 2005) and their coevolution. Indeed, AGN feedback can shut down the growth of the most massive systems, steepening the bright-end slope of the LF (Scannapieco \& Oh 2004), while the feedback-driven QSO decay determines the shape of the faint-end slope (Hopkins et al. 2006).

This evolutionary trend has not been clearly seen yet in optically selected type-1 AGN samples. By combining results from low and high redshifts, it is clear from the studies of optically selected samples that the cosmic QSO evolution shows a strong increase of the activity from $z \sim 0$ out to $z \sim 2$, reaches a maximum around $z \simeq 2-3$ and then declines, but the shape of the turnover and the redshift evolution of the peak in activity as a function of luminosity is still unclear.

Most of the optically selected type-1 AGN samples studied so far are obtained through various color selections of candidates, followed by spectroscopic confirmation (e.g. $2 \mathrm{dF}$, Croom et al. 2004 and SDSS, Richards et al. 2002), or grism and slitless spectroscopic surveys. These samples are expected to be highly complete, at least for luminous type-1 AGN, at either $z \leq 2.2$ or $z \geq 3$.6, where type-1 AGN show conspicuous colors in broad-band color searches, but less complete in the redshift range $2.2 \leq z \leq 3.6$ (Richards et al. 2002).

An improvement in the multi-color selection in the optical can be achieved through the simultaneous use of many broadand medium-band filters, such as those used in the COMBO-17 survey (Wolf et al. 2003). This survey is the only optical survey so far which, in addition to reaching redshifts beyond the peak of AGN activity, is also deep enough to sample up to high redshift type-1 AGN with luminosities below the break in the luminosity function. However, only photometric redshifts are available for this sample and, because of their selection criteria, it is incomplete for objects with small nuclear flux compared to the total host-galaxy emission, and for AGN with anomalous colors, such as, for example, the broad absorption-line (BAL) QSOs, which have on average redder colors and account for $\sim 10-15 \%$ of the overall AGN population (Hewett \& Foltz 2003).

The VIMOS-VLT Deep Survey (Le Fèvre et al. 2005) is a spectroscopic survey in which the target selection is purely fluxlimited (in the $I$-band), with no additional selection criterion. This allows the selection of a spectroscopic type-1 AGN sample free of color and/or morphological biases above redshift $z>1$. An obvious advantage of such a selection is the possibility to test the completeness of the most current surveys (see Gavignaud et al. 2006, Paper I), which make use of morphological and/or color pre-selections, and to study the evolution of type-1 AGN activity in a large redshift range.

In this paper we use the type-1 AGN sample selected from the VVDS to determine the luminosity function in the redshift range $1<z<3.6$. The VVDS type-1 AGN sample is more than one magnitude deeper than any previous optically-selected sample and thus allows to explore the faint part of the luminosity function. Moreover, by combining this LF with measurement of the LF in much larger, but very shallow, surveys, we can derive an analytical form to describe the evolution of type-1 AGN in the redshift range $0<z<4$ in a large luminosity range. The paper is organized as follows: in Sects. 2 and 3 we describe the sample and its color properties. In Sect. 4 we present the method used to determine the luminosity function, while in Sect. 5 we compare it with previous works both at low and high redshifts. The bolometric LF and the comparison with the results derived from samples selected in different bands (from X-ray to IR) is then presented in Sect. 6. The derived LF-fitting models are presented in Sect. 7, while the AGN activity as a function of redshift is presented in Sect. 8. Finally in Sect. 9 we summarize our results. Throughout this paper, unless stated otherwise, we assume a cosmology with $\Omega_{\mathrm{m}}=0.3, \Omega_{\Lambda}=0.7$ and $H_{0}=70 \mathrm{~km} \mathrm{~s}^{-1} \mathrm{Mpc}^{-1}$.

\section{The sample}

Our AGN sample is built from the first-epoch data of the VIMOS-VLT Deep Survey (Le Fèvre et al. 2005). The VVDS is a spectroscopic survey designed to measure about 150000 redshifts of galaxies in the redshift range $0<z<5$, selected nearly randomly from a dedicated $I$-band imaging survey (which is complemented by observations in $U, B, V$ and $R$ bands, as well as, for part of the field, in the $K$ band). Full details about VVDS photometry can be found in Le Fèvre et al. (2004a), McCracken et al. (2003), Radovich et al. (2004) for the $U$-band and Iovino et al. (2005) for the $K$-band. In this work we also use the Galex $U V$-catalog (Arnouts et al. 2005; Schiminovich et al. 2005), the $u^{*}, g^{\prime}, r^{\prime}, i^{\prime}, z^{\prime}$ photometry obtained in the frame of the CanadaFrance-Hawaii Legacy Survey (CFHTLS) ${ }^{1}$, UKIDSS (Lawrence et al. 2006) and the Spitzer Wide-area InfraRed Extragalactic survey (SWIRE) (Lonsdale et al. 2003, 2004). The spectroscopic VVDS survey consists of a deep and a wide survey, with a very simple selection function relying solely on the $I$-band magnitude: $17.5<I_{\mathrm{AB}}<22.5$ for the wide and $17.5<I_{\mathrm{AB}}<24.0$

\footnotetext{
1 wWw.cfht.hawaii.edu/Science/CFHLS
} 


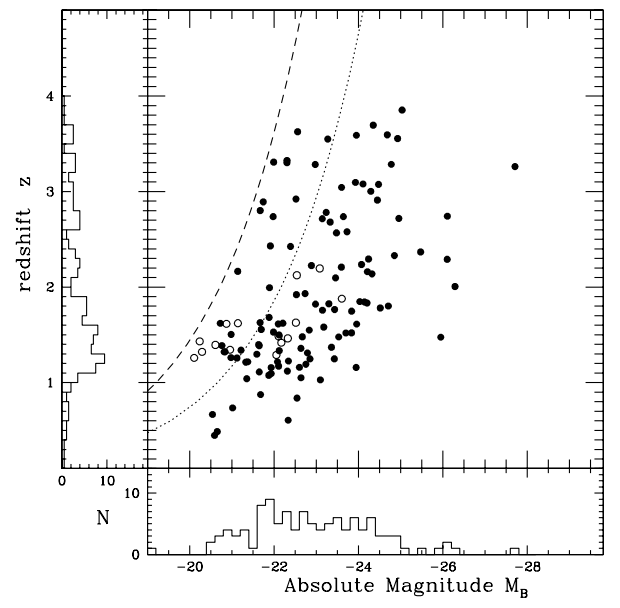

Fig. 1. Distribution of absolute magnitudes and redshifts of the total AGN sample. Open circles are the objects with ambiguous redshift, shown at all their possible $z$ values. The dotted and dashed lines represent the magnitude limits of the samples: $I_{\mathrm{AB}}<22.5$ for the wide sample and $I_{\mathrm{AB}}<24.0$ for the deep sample.

for the deep sample. For a detailed description of the spectroscopic survey strategy and the first epoch data see Le Fèvre et al. (2005).

Our sample consists of 130 AGN with $0<z<5$, selected in 3 VVDS fields $(0226-04,1003+01$ and 2217-00) and in the Chandra Deep Field South (CDFS, Le Fèvre et al. 2004b), the 0226-04 and the CDFS fields being part of the deep survey and the 1003+01 and 2217-00 fields of the wide survey. All of them are selected as AGN on the basis of the presence of at least one broad emission line in their spectra, irrespectively of their morphological or color properties. We discovered 74 of them in the deep fields (62 in the 0226-04 field and 12 in the CDFS) and 56 in the wide fields ( 18 in the $1003+01$ field and 38 in the $2217-00$ field). This represents an unprecedented complete sample of faint AGN, free of morphological or color selection bias. The spectroscopic area covered by the first-epoch data is $0.62 \mathrm{deg}^{2}$ in the deep fields (0226-04 field and CDFS) and $1.1 \mathrm{deg}^{2}$ in the wide fields (1003+01 and 2217-00 fields).

To each object we have assigned a redshift and a spectroscopic quality flag which quantifies our confidence level in that given redshift. We have $115 \mathrm{AGN}$ with secure redshifts, and 15 AGN with two or more possible values, because only one broad emission line, with no other narrow lines and/or additional features, is detected in the VIMOS spectral range (5500-9500 A) (see Fig. 1 in Paper I). For all of them, however, a best solution is proposed. In the original VVDS AGN sample, the number of AGN with this redshift degeneracy was 42 . This number has been reduced first by searching for objects already observed in other spectroscopic surveys, solving the redshift for 3 of them. For the remaining objects, we performed a spectroscopic follow-up with FORS1 on the VLT Unit Telescope 2 (UT2). With these additional observations we found a secure redshift for 24 of our AGN with ambiguous redshift determination; in addition, we found that our proposed best solution was the correct one in $\sim 80 \%$ of the cases. On the basis of this result, in the following analysis the best estimate of the redshift will be used for the 15 remaining AGN with ambiguous redshift determination.

In Fig. 1 we show the absolute B-magnitude and redshift distributions of the sample. As shown in this figure, our sample spans a large range of luminosities and consists of both Seyfert

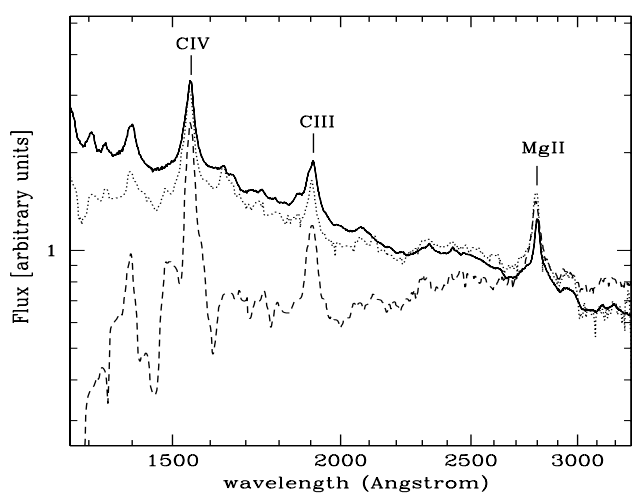

Fig. 2. Composite spectra derived for our AGN with secure redshift in the 0226-04 field, divided in a "bright" (19 objects at $M_{1450}<-22.15$, dotted curve) and a "faint" ( 31 objects at $M_{1450}>-22.15$, dashed curve) subsample. We consider here only AGN with $z>1$. The SDSS composite spectrum is shown with a solid line for comparison.

galaxies $\left(M_{B}>-23 ; \sim 59 \%\right)$ and QSOs $\left(M_{B}<-23 ; \sim 41 \%\right)$. A more detailed and exhaustive description of the properties of the AGN sample is given in Paper I (Gavignaud et al. 2006) and the complete list of broad-line AGN in our wide and deep samples is available as an electronic table in the appendix of Gavignaud et al. (2006).

\section{Colors of type-1 AGN}

As already discussed in Paper I, the VVDS AGN sample shows, on average, redder colors than those that can be derived from the SDSS composite spectrum (Vanden Berk et al. 2001). In Paper I we proposed three possible explanations: (a) the contamination of the host galaxy is reddening the observed colors of faint AGN; (b) type-1 AGN are intrinsically redder when they are faint; (c) the redder colors are due to dust extinction. On the basis of the statistical properties of the sample, we concluded that hypothesis (a) was likely to be the more correct, as expected from the faint absolute magnitudes sampled by our survey, even if hypotheses (b) and (c) could not be ruled out.

In Fig. 2 we show the composite spectra derived from the sample of AGN with secure redshift in the 0226-04 field, divided in a "bright" and a "faint" sample at the absolute magnitude $M_{1450}=-22.15$. We consider here only AGN with $z>1$, which corresponds to the AGN used in Sect. 4 to compute the luminosity function. The choice of the reference rest-frame wavelength for the absolute magnitude, $\lambda=1450 \AA$, is motivated by our photometric coverage. In fact, for most of the objects it is possible to interpolate $M_{1450}$ directly from the observed magnitudes. In the same plot we show also the SDSS composite spectrum (solid curve) for comparison. Even if the "bright" VVDS composite (dotted curve) is already somewhat redder than the SDSS one, it is clear from this plot that the main difference occurs for the faintest objects (dashed curve).

A similar result is shown for the same sample in the upper panel of Fig. 3, where we plot the spectral index $\alpha$ as a function of the AGN luminosity. The spectral index is derived here by fitting a simple power law $f(v)=v^{-\alpha}$ to our photometric data points. This analysis has been performed only on the 0226-04 deep sample, since for the wide sample we do not have enough photometric coverage to reliably derive the spectral index. Most of the AGN with $\alpha>1$ are fainter than $M_{1450}=-22.15$, showing that, indeed, the faintest objects have on average redder 


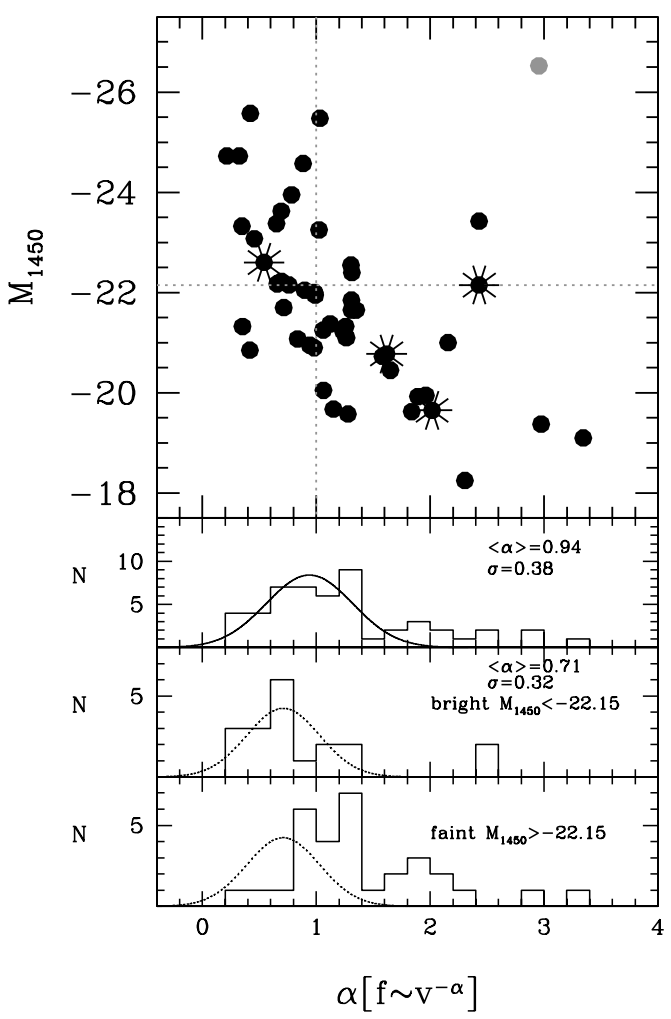

Fig. 3. Upper panel: distribution of the spectral index $\alpha$ as a function of $M_{1450}$ for the same sample of AGN as in Fig. 2. Asterisks are AGN morphologically classified as extended and the grey point is a BAL AGN. Bottom panels: distribution of the spectral index $\alpha$ for the same sample of AGN. All the AGN in this sample are shown in the first of the three panels, while the AGN in the "bright" and "faint" sub-samples are shown in the second and third panel, respectively. The dotted curve in the second and third panels corresponds to the Gaussian fit of the bright sub-sample.

colors than the brightest ones. The outlier (the brightest object with large $\alpha$, i.e. very red colors, in the upper right corner of the plot) is a BAL AGN.

The three bottom panels of Fig. 3 show the histograms of the resulting power-law slopes for the same AGN sample. A Gaussian curve with $\langle\alpha\rangle=0.94$ and standard deviation $\sigma=0.38$ is a good representation for the distribution of about $80 \%(40 / 50)$ of the objects in the first panel. In addition, there is a significant tail $(\sim 20 \%)$ of redder AGN with slopes in the range from 1.8 up to $\sim 3.0$. The average slope of the total sample $(\sim 0.94)$ is redder than the fit to the SDSS composite $(\sim 0.44)$. Moreover, the distribution of $\alpha$ is shifted toward much larger values (redder continua) than the similar distribution in the SDSS sample (Richards et al. 2003). For example, only 6\% of the objects in the SDSS sample have $\alpha>1.0$, while this percentage is $57 \%$ in our sample.

The differences with respect to the SDSS sample can be partly due to the differences in absolute magnitude of the two samples $\left(M_{i}<-22.0\right.$ for the SDSS sample (Schneider et al. 2003) and $M_{B}<-20.0$ for the VVDS sample). In fact, if we consider the VVDS "bright" sub-sample, the average spectral in$\operatorname{dex}\langle\alpha\rangle$ becomes $\sim 0.71$, which is closer to the SDSS value (even if it is still somewhat redder), and only two objects ( $\sim 8 \%$ of the sample) show values not consistent with a Gaussian distribution with $\sigma \sim 0.32$. Moreover, only 30\% of this sample have $\alpha>1.0$.

Most of the bright SDSS AGN with $\alpha>1$ are interpreted by Richards et al. (2003) to be dust-reddened, although a fraction

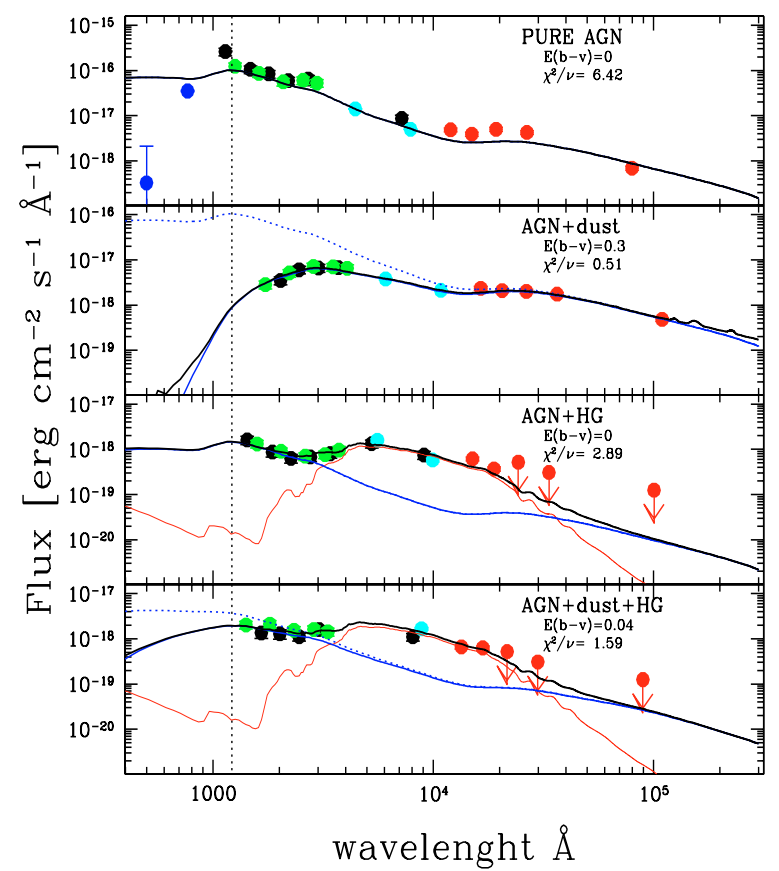

Fig. 4. Four examples of different decompositions of the observed SEDs of our objects. Photometric data below $\lambda<1216 \AA$ in the rest-frame are considered lower-limits. The four fits shown in this figure correspond, from top to bottom, to pure-AGN, dust-extincted AGN, AGN and host galaxy, dust-extincted AGN and host galaxy. The dotted line corresponds to the AGN template before the application of the extinction law, while the solid blue line corresponds to the same template taking extinction into account. The red line (third and fourth panel) corresponds to the galaxy template and, finally, the black line is the resulting best fit to the SED. Arrows correspond to $5 \sigma$ upper limits in case of non detection in the IR.

of them is likely to be due to intrinsically red AGN (Hall et al. 2006). At fainter magnitude one would expect both a larger fraction of dust-reddened objects (in analogy with indications from the X-ray data, Brandt et al. 2000; Mushotzky et al. 2000) and a more significant contamination from the host galaxy.

We have tested these possibilities by examining the global spectral energy distribution (SED) of each object and by fitting the observed fluxes $f_{\text {obs }}$ with a combination of AGN and galaxy emission, allowing also for the possibility of extinction of the AGN flux. Thanks to the multi-wavelength coverage in the deep field, for which we have, in addition to VVDS bands, data from GALEX, CFHTLS, UKIDSS and SWIRE, we can study the spectral energy distribution of the single objects. In particular, we assume that:

$f_{\mathrm{obs}}=c_{1} f_{\mathrm{AGN}} \cdot 10^{-0.4 \cdot A_{\lambda}}+c_{2} f_{\mathrm{GAL}}$.

Using a library of galaxy and AGN templates, we find the best parameters $c_{1}, c_{2}$ and $E_{B-V}$ for each object. We used the AGN SED derived by Richards et al. (2006a) with an SMC-like dustreddening law (Prevot et al. 1984) with the form $A_{\lambda} / E_{B-V}=$ $1.39 \lambda_{\mu \mathrm{m}}^{-1.2}$, and a library of galaxy templates by Bruzual \& Charlot (2003). Since the observed flux below the Ly $\alpha$ line is affected by intervening absorption, all the photometric data at rest-frame wavelengths $\lambda<1216 \AA$ are treated as lower-limits in the fits.

We found that for $\sim 37 \%$ of the objects, the observed flux is fitted by a typical AGN power-law (pure AGN), while $44 \%$ of the sources require the presence of a contribution from the host galaxy to reproduce the observed flux. Only $4 \%$ of the objects 
are fitted by pure AGN + dust, while the remaining $15 \%$ of the objects require both the contribution of the host galaxy contamination and the presence of dust. As expected, if we restrict the analysis to the bright sample, the percentage of pure AGN increases to $68 \%$, with the rest of the objects requiring either some contribution from the host galaxy $(\sim 21 \%)$ or the presence of dust obscuration $(\sim 11 \%)$.

In Fig. 4 we show 4 examples of the resulting fits: (i) pure AGN; (ii) dust-extincted AGN; (iii) AGN contaminated by the host galaxy; (iv) dust-extincted AGN and contaminated by the host galaxy. The dotted line corresponds to the AGN template before applying the extinction law, while the solid blue line corresponds to the same template with extinction; the red line corresponds to the galaxy template and, finally, the black line is the resulting best fit to the SED. The host galaxy contaminations will be taken into account in the computation of the AGN absolute magnitude for the luminosity function.

\section{Luminosity function}

\subsection{Definition of the redshift range}

For the study of the LF we decided to exclude AGN with $z \leq 1.0$. This choice is due to the fact that for $0.5 \leq z \leq 1.0$ the only visible broad line in the VVDS spectra is $\mathrm{H} \beta$ (see Fig. 1 of Paper I). This means that all objects with narrow or almost narrow $\mathrm{H} \beta$ and broad $\mathrm{H} \alpha$ (type 1.8, 1.9 AGN; see Osterbrock 1981) would not be included in our sample. Since at low luminosities the number of intermediate type AGN is not negligible, this redshift bin is likely to be under-populated and the results would not be meaningful.

At $z<0.5$, in principle we have less problems, because $\mathrm{H} \alpha$ is within the wavelength range of the VVDS spectra, but at this low redshift our sampled volume is relatively small. Because of the rarity of QSOs, we have only 3 objects with secure redshifts in this redshift bin in the current sample. For these reasons, our luminosity function has been computed only for $z>1.0$ AGN. As already mentioned in Sect. 2, the small fraction of objects with an ambiguous redshift determination has been included in the computation of the luminosity function assuming that our best estimate of their redshift is correct.

The resulting sample used in the computation of the LF consists therefore of 121 objects at $1<z<4$.

\subsection{Incompleteness function}

Our incompleteness function is made up of two terms linked, respectively, to the selection algorithm and to the spectral analysis: the target sampling rate (TSR) and the spectroscopic success rate (SSR) defined following Ilbert et al. (2005).

The TSR is the ratio between the number of observed sources and the total number of objects in the photometric catalog with magnitudes inside the range of the VVDS. It gives the probability that a source that fulfills the criteria for being a potential target has actually been observed. The TSR is similar in the wide and deep sample and runs from $20 \%$ to $30 \%$ depending on the apparent size of the object.

The SSR is the probability of a spectroscopically targeted object to be securely identified. It is a complex function of the redshift, apparent magnitude and intrinsic spectral energy distribution. It has been estimated by simulating 20 VIMOS pointings, for a total of 2745 AGN spectra (see Paper I).

Full details on TSR and SSR can be found in Paper I (Gavignaud et al. 2006). They are taken into account in the computation of the weight associated to each object $w^{\text {object }}=$ $w^{\text {tsr }} \cdot w^{\text {ssr }}=1 / T S R \cdot 1 / S S R$.

\subsection{Estimation of the absolute magnitude}

We derive the absolute magnitude in the reference band using the apparent magnitude in the observed band:

$M=m_{\mathrm{obs}}-5 \log _{10}(\mathrm{~d} l(z))-25-k$

where $M$ is computed in the band in which we want to compute the luminosity function, $m_{\mathrm{obs}}$ is the observed band from which we want to calculate it, $\mathrm{d} l(z)$ is the luminosity distance expressed in $\mathrm{Mpc}$ and $k$ is the $\mathrm{k}$-correction in the reference band. To make easier the comparison with previous results in the literature, we computed the luminosity function in the $B$-band.

We compute the absolute magnitudes using the template derived from the SDSS sample (Vanden Berk et al. 2001). To minimize the uncertainties in the adopted k-correction, $m_{\mathrm{obs}}$ for each object should be chosen in the observed band closest to the restframe band in which the luminosity function is computed. The best bands to compute the $B$-band absolute magnitudes are in the infrared as soon as $z \gtrsim 2$. Unfortunately, the $I$ band is the reddest band that is available for all our objects; we decided to use it for all objects to compute the $B$-band magnitudes. This means that for $z \gtrsim 2$ we introduce an additional uncertainty in the absolute magnitudes due to the k-correction.

As discussed in Sect. 3, the VVDS AGN sample shows redder colors than those typical of normal, more luminous AGN, which can be due to the combination of the host-galaxy contribution and the presence of dust. Since, in this redshift range, the fractional contribution from the host galaxies is expected to be more significant in the I-band than in bluer bands, the luminosity derived using the $I$-band observed magnitude could, in some cases, be somewhat overestimated.

We estimated the possible impact of this effect on our results in the following way. From the results of the analysis of the SED of the single objects in the deep sample (see Sect. 3) we computed for each object the difference $m_{I}(\mathrm{TOT})-m_{I}(\mathrm{AGN})$ and, consequently, $M_{B}$ (TOT) $-M_{B}(\mathrm{AGN})$. This allows us to derive the LF using the derived $M_{B}(\mathrm{AGN})$, resolving the possible bias introduced by the host-galaxy contamination. These differences are shown in Fig. 5 as a function of absolute magnitude (upper panel) and redshift (lower panel). For most objects the resulting differences are small $(\Delta M \leq 0.2)$. However, for a non-negligible fraction of the faintest objects $\left(M_{B} \geq-22.5, z \leq 2.0\right)$ these differences can be significant (up to $\sim 1 \mathrm{mag}$ ). For the wide sample, for which the more restricted photometric coverage does not allow detailed SED analysis and decomposition, $M_{B}(\mathrm{AGN})$ is derived using a Monte-Carlo simulation based on the bivariate distribution $\delta(M, z)$ estimated from the objects in the deep sample, $\delta(M, z)$ being the probability distribution of $\Delta M$ as a function of $M_{B}$ and $z$. The resulting simulated differences for the objects in the wide sample are also shown in Fig. 5.

The AGN magnitudes and the limiting magnitudes of the samples have been corrected for galactic extinction on the basis of the mean extinction values $E(B-V)$ in each field derived from Schlegel et al. (1998), except for the 2217-00 field, where the extinction is highly variable across the field, and where we used a different extinction for each individual objects. The resulting corrections in the $I$-band magnitude are $A_{I} \simeq 0.027$ in the 0226-04 and 1003+01 fields and $A_{I}=0.0089$ in the CDFS field, while the average value in the 2217-00 field is $A_{I}=0.065$. 

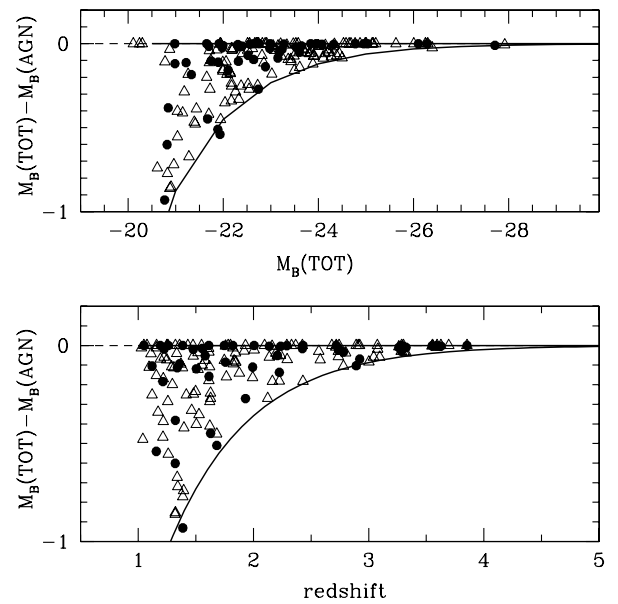

Fig. 5. Real (full circles; AGN in the deep sample) and simulated (open triangles; AGN in the wide sample) $B$-band absolute-magnitude differences as a function of $M_{B}$ (TOT) (upper panel) and redshift (bottom panel). $M_{B}$ (TOT) is the absolute magnitude computed considering the total observed flux, while $M_{B}(\mathrm{AGN})$ is the absolute magnitude computed after subtracting the host-galaxy contribution.

\subsection{The $1 / V_{\max }$ estimator}

We derive the binned representation of the luminosity function using the usual $1 / V_{\max }$ estimator (Schmidt 1968), which gives the space-density contribution of individual objects. The luminosity function for each redshift bin $(z-\Delta z / 2 ; z+\Delta z / 2)$ is then computed as:

$\Phi(M)=\frac{1}{\Delta M} \sum_{M-\Delta M / 2}^{M+\Delta M / 2} \frac{w_{i}^{\mathrm{tsr}} w_{i}^{\mathrm{ssr}}}{V_{\max , i}}$

where $V_{\max , i}$ is the comoving volume within which the $i$ th object would still be included in the sample. The statistical uncertainty on $\Phi(\mathrm{M})$ is given by Marshall et al. (1983):

$\sigma_{\phi}=\frac{1}{\Delta M} \sqrt{\sum_{M-\Delta M / 2}^{M+\Delta M / 2} \frac{\left(w_{i}^{\mathrm{tsr}} w_{i}^{\mathrm{ssr}}\right)^{2}}{V_{\max , i}^{2}}}$

We combine our samples at different depths using the method proposed by Avni \& Bahcall (1980). This method is based on the fact that each object characterized by an observed redshift $z_{i}$ and intrinsic luminosity $L_{i}$ could have been found in any of the survey areas for which its observed magnitude is brighter than the corresponding magnitude limit. This means that, for our total sample, we consider an area of:

$\Omega_{\mathrm{tot}}(m)=\Omega_{\text {deep }}+\Omega_{\text {wide }}=1.72 \mathrm{deg}^{2}$ for $17.5<I_{\mathrm{AB}}<22.5$ and

$\Omega_{\mathrm{tot}}(m)=\Omega_{\text {deep }}=0.62 \mathrm{deg}^{2} \quad$ for $\quad 22.5<I_{\mathrm{AB}}<24.0$.

The resulting luminosity functions in different redshift ranges are shown in Figs. 6 and 7, while the details for each bin are presented in Table 1. These values are not corrected for the host galaxy contribution. We have verified that, even if the differences between the total absolute magnitudes and the magnitudes corrected for the host galaxy contribution (see Sect. 4.3) can be significant for a fraction of the faintest objects, the resulting luminosity functions computed using these two sets of absolute magnitudes are not significantly different. For this reason and for a more direct comparison with previous works, the results on the luminosity function presented in the next section are those obtained using the total magnitudes.

\section{Comparison with the results from other optical surveys}

\subsection{The low redshift luminosity function}

In Fig. 6 we present our luminosity function in two redshift bins, $1.0<z<1.55$ and $1.55<z<2.1$, compared to the LF fits derived from the 2dF QSO sample (Croom et al. 2004; Boyle et al. 2000), with the COMBO-17 sample (Wolf et al. 2003) and with the 2dF-SDSS (2SLAQ) LF fit (Richards et al. 2005). In each panel the curves correspond to a broken powerlaw luminosity function in which the redshift evolution is characterized by a pure luminosity evolution modeled as $M_{b}^{*}(z)=$ $M_{b}^{*}(0)-2.5\left(k_{1} z+k_{2} z^{2}\right)$. Moreover, the thick parts of the curves show the luminosity range covered by the data in each of the comparison samples, while the thin parts are extrapolation based on the best fit parameters of the models.

As shown in Fig. 6, even if our LF, given the covered luminosity range, don't show any break in both redshift intervals, our bright LF data points connect rather smoothly to the faint part of the $2 \mathrm{dF}$ data. However, our sample is more than two magnitudes deeper than the $2 \mathrm{dF}$ sample. For this reason, a comparison at low luminosity is possible only with the extrapolations of the LF fit. At $z>1.55$, while the Boyle's model fits well our faint LF data points, the Croom's extrapolation, being very flat, tends to underestimate our low luminosity data points. At $z<1.55$ the comparison is worse: as in the higher redshift bin, the Boyle's model fits our data better than the Croom's one but, in this redshift bin, our data points show an excess at low luminosity with respect to Boyle's fit. This trend is similar to that shown by the comparison with the fit to the COMBO-17 data, which, contrarily to the $2 \mathrm{dF}$ data, have a low-luminosity limit close to ours: at $z>1.55$ the agreement is very good, but in the first redshift bin our data show again an excess at low luminosity. This excess is likely due to the fact that, because of its selection criteria, the COMBO-17 sample is expected to be significantly incomplete for objects in which the ratio between the nuclear flux and the total host galaxy flux is small. Finally, we compare our data with the 2SLAQ fits derived by Richards et al. (2005). The 2SLAQ data are derived from a sample of AGN selected from the SDSS with magnitude $18.0<g<21.85$ and $z<3$, and observed with the 2-degree field instrument. Similarly to the $2 \mathrm{dF}$ sample, the LF is derived only for $z<2.1$ and $M_{B}<-22.5$. The plotted dot-dashed curve corresponds to a PLE model in which most of the model parameters are fixed at the values found by Croom et al. (2004), leaving as free parameters the faint-end slope and the normalization constant $\Phi^{*}$ only. In this case, the agreement with our data points at $z<1.55$ is very good even at low luminosity. The faint end slope found in this case is $\beta=-1.45$, which is similar to that found by Boyle et al. (2000) $(\beta=-1.58)$ and significantly steeper than that found by Croom et al. (2004) $(\beta=-1.09)$. At $z>1.55$, the Richards et al. (2005) LF fit tends to overestimate our data points at the faint end of the LF, which suggests a flatter slope in this redshift bin.

The first conclusion from this comparison is that, at low redshift (i.e. $z<2.1$ ), the data from our sample, which is $\sim 2$ mag fainter than the previous spectroscopically confirmed samples, are not well fitted simultaneously in the two analyzed redshift bins by the PLE models derived from previous samples. Qualitatively, the main reason for this appears to be the fact that our data suggest a change in the LF faint-end slope, which appears to flatten with increasing redshift. This trend, already observed in previous X-ray surveys (La Franca et al. 2002; Ueda et al. 2003; Fiore et al. 2003; La Franca et al. 2005) suggests 


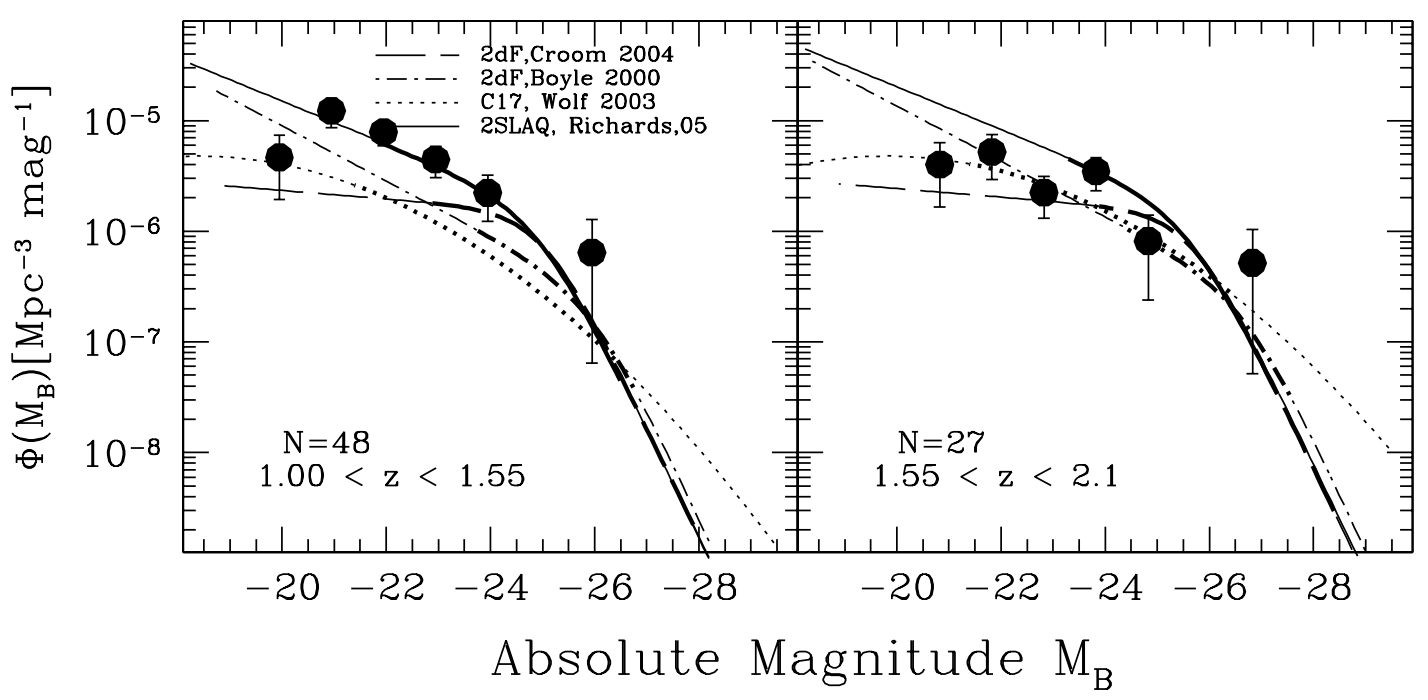

Fig. 6. Rest-frame $B$-band luminosity function derived in the redshift bins $1.0<z<1.55$ and $1.55<z<2.1$, compared to the $2 \mathrm{dFQRS}$ (Croom et al. 2004; Boyle et al. 2000), COMBO-17 (Wolf et al. 2003) and 2dF-SDSS (2SLAQ Richards et al. 2005) data. The curves in the figure show the PLE fit models from these authors. The thick parts of the curves correspond to the luminosity range covered by the data in each sample, while the thin parts are extrapolations based on the best fit parameters.

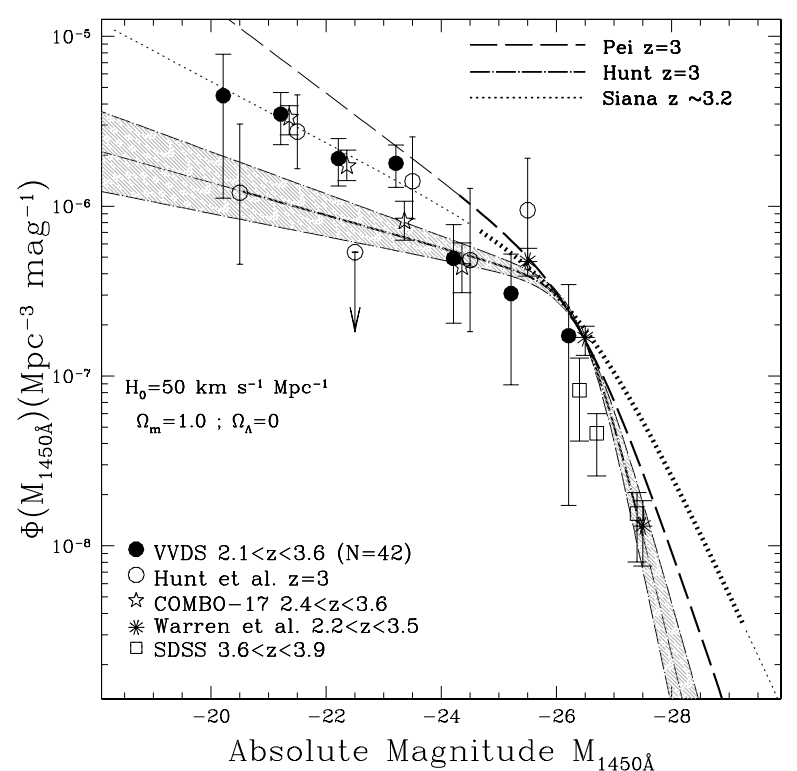

Fig. 7. The $1450 \AA$ rest-frame luminosity function in the redshift range $2.1<z<3.6$ compared to data from other high-redshift samples (Hunt et al. (2004) at $z=3$; Combo-17 data from Wolf et al. (2003) at $2.4<$ $z<3.6$; data from Warren et al. (1994) at $2.2<z<3.5$ and the SDSS data from Fan et al. (2001)). The SDSS data points at $3.6<z<3.9$ have been evolved to $z=3$ using the luminosity evolution of Pei (1995) as in Hunt et al. (2004). The thick lines show model fits within the luminosity range covered by the samples, while the thin lines are model extrapolation. For this plot, an $\Omega_{\mathrm{m}}=1, \Omega_{\Lambda}=0, h=0.5$ cosmology has been assumed for comparison with previous works.

that a simple PLE parameterization may not be a good representation of the evolution of the AGN luminosity function over a wide range of redshifts and luminosities. Different model fits will be discussed in Sect. 7.

\subsection{The high-redshift luminosity function}

The comparison of our LF data points for $2.1<z<3.6$ (full circles) with the results from other samples in similar redshift ranges is shown in Fig. 7. In this figure an $\Omega_{\mathrm{m}}=1, \Omega_{\Lambda}=0$, $h=0.5$ cosmology has been assumed for comparison with previous works, and the absolute magnitude has been computed at $1450 \AA$. In terms of number of objects, depth and covered area, the only sample comparable to ours is the COMBO-17 sample (Wolf et al. 2003), which, in this redshift range, consists of 60 AGN candidates over 0.78 square degree. At a similar depth we show also the data from the sample of Hunt et al. (2004), which consists of 11 AGN in the redshift range $\langle z\rangle \pm \sigma_{z}=3.03 \pm 0.35$ (Steidel et al. 2002). Given the small number of objects, the corresponding Hunt model fit was derived including the Warren data points (Warren et al. 1994). Moreover, they assumed the Pei (1995) luminosity evolution model, adopting the same values for $L^{*}$ and $\Phi^{*}$, leaving the two faint- and bright-end slopes free. For comparison we also show the original Pei model fit derived from the empirical luminosity function estimated by Hartwick \& Schade (1990) and Warren et al. (1994). In the same plot we also show the model fit derived from a sample of $\sim 100 z \sim 3$ (U-dropout) QSO candidates by Siana et al. (private comunication; see also Siana et al. 2006). This sample has been selected using a simple optical/IR photometric selection at $19<r^{\prime}<22$, and the bright-end slope was fixed to that determined at $z=-2.85$ using SDSS data (Richards et al. 2006b).

In general, the VVDS LF is in agreement with those from other surveys in the region of overlapping magnitudes. The best agreement is with the Siana model with a faint end slope $\beta=-1.45$. It is interesting to note that, in the faint part of the LF, our data points appear to be higher with respect to the Hunt et al. (2004) fit and are instead closer to the extrapolation of the original Pei model fit. This difference is probably due to the fact that, having only 11 AGN in their faint sample, their faint-end slope was poorly constrained.

\section{The bolometric luminosity function}

The comparison between the AGN LFs derived from samples selected in different bands has been for a long time a critical point in the studies of the AGN luminosity function. Recently, Hopkins et al. (2007), combining a large number of LF 


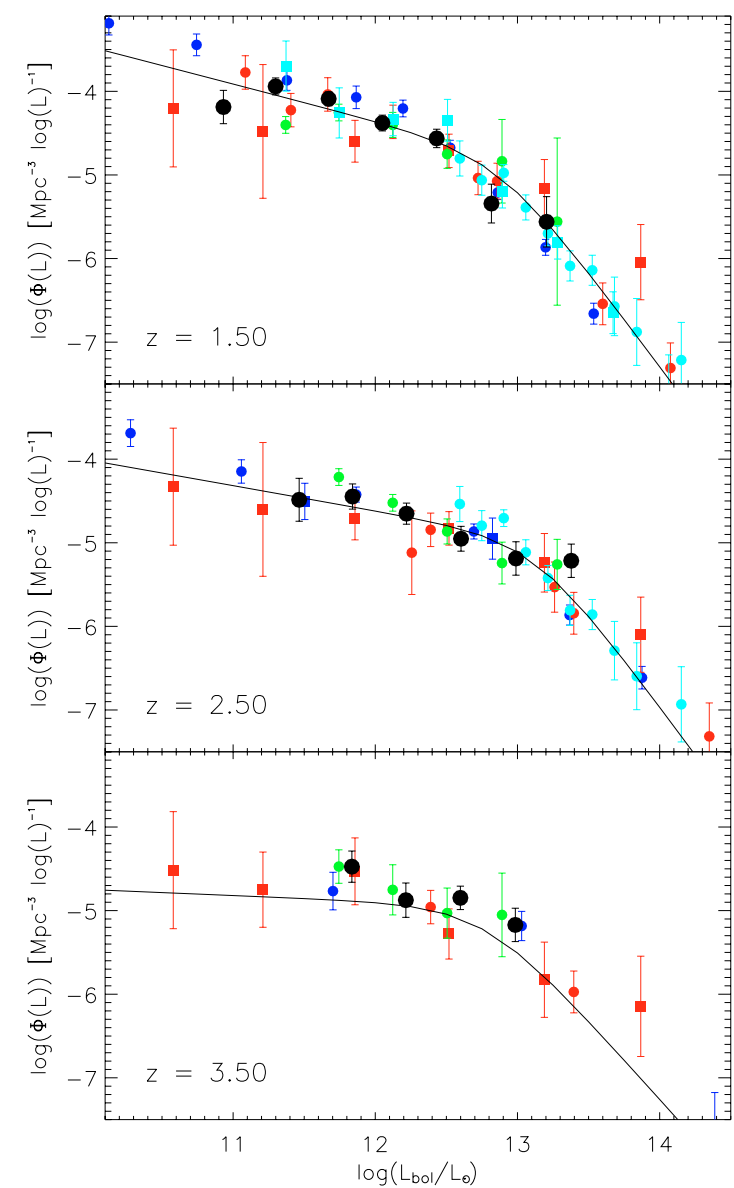

Fig. 8. Bolometric luminosity function derived in three redshift bins from our data (black dots), compared with Hopkins et al. (2007) bestfit model and the data-sets used in their work. Red symbols correspond to hard X-ray surveys (squares: Barger et al. 2005; circles: Ueda et al. 2003). Blue to soft X-ray surveys (squares: Silverman et al. 2005; circles: Hasinger et al. 2005). Cyan to infra-red surveys (circles: Brown et al. 2006; squares: Matute et al. 2006). The green circles are the COMBO-17 optical surveys (Wolf et al. 2003).

measurements obtained in different redshift ranges, observed wavelength bands and luminosity intervals, derived the bolometric QSO luminosity function in the redshift range $z=0-6$. For each observational band, they derived appropriate bolometric corrections, taking into account the variation with luminosity of both the average absorption properties (e.g. the $N_{\mathrm{H}}$ column density from X-ray data) and the average global spectral energy distributions. They show that, with these bolometric corrections, it is possible to find a good agreement between results from all different data sets.

We applied the bolometric corrections given by Eqs. (2) and (4) of Hopkins et al. (2007) for the $B$-band to our LF data points. The bolometric LF is shown in Fig. 8, together with the bolometric-LF best-fit model of Hopkins et al. (2007). Bolometric LFs derived by applying the same procedure to the LFs from other surveys are also presented.

Our data, which sample the faint part of the bolometric luminosity function better than all previous optically selected samples, are in good agreement with all the other samples, selected in different bands. Only in the last redshift bin, our data are quite higher with respect to them. The agreement remains however good with the COMBO-17 sample, which is the only optically selected sample plotted here. This effect may be due to the fact that the conversions used to compute the bolometric LF have been derived for AGN at low redshift and become less accurate at high redshift.

Our data show good agreement also with the model fit derived by Hopkins et al. (2007). By trying various analytical fits to the bolometric luminosity function, Hopkins et al. (2007) concluded that neither pure luminosity nor pure density evolution represent well all the data. An improved fit can instead be obtained with a luminosity dependent density evolution model (LDDE) or, even better, with a PLE model in which both the bright- and the faint-end slopes evolve with redshift. Both these models can reproduce the observed flattening with redshift of the faint end of the luminosity function.

\section{Model fitting}

In this section we discuss the results of a number of different fits to our data as a function of luminosity and redshift. For this purpose, we compute the luminosity function in 5 redshift bins between $z=1.0$ and $z=4.0$. Since, in this redshift range, our data cover only the faint part of the luminosity function, we complement our data with the LF data points from the SDSS data release 3 (DR3) (Richards et al. 2006b). The SDSS sample has the advantage over, for example, the $2 \mathrm{dF}$ sample of probing the luminosity function to much higher redshifts. The SDSS sample contains more than 15000 spectroscopically confirmed AGN selected from an effective area of 1622 sq. deg. Its limiting magnitude $(i<19.1$ for $z<3.0$ and $i<20.2$ for $z>3.0$ ) is much brighter than the VVDS, and therefore does not sample well the the faint end of the AGN luminosity function. For this reason, Richards et al. (2006b) fitted the SDSS data using a single power-law to describe the luminosity function above the break luminosity. Adding the VVDS data, which mainly sample the faint end, allows us to cover the entire luminosity range in the common redshift range $(1.0<z<4.0)$, also extending the analysis at $z<1.0$ where only SDSS data are available.

For all the analyzed models we have parameterized the luminosity function as a double power-law that, expressed in luminosity, is given by:

$\Phi(L, z)=\frac{\Phi_{L}^{*}}{\left(L / L^{*}\right)^{-\alpha}+\left(L / L^{*}\right)^{-\beta}}$

where $\Phi_{L}^{*}$ is the characteristic AGN density in $\mathrm{Mpc}^{-3}, L^{*}$ is the characteristic luminosity and $\alpha$ and $\beta$ are the two power-law indices. Eq. (5) can be expressed in absolute magnitude as:

$\Phi(M, z)=\frac{\Phi_{M}^{*}}{10^{0.4(\alpha+1)\left(M-M^{*}\right)}+10^{0.4(\beta+1)\left(M-M^{*}\right)}}$.

\subsection{The PLE and PDE models}

The first model that we tested is a pure luminosity evolution (PLE) with the following evolution in redshift:

$M^{*}(z)=M^{*}(0)-2.5\left(k_{1} z+k_{2} z^{2}\right)$.

Following Richards et al. (2006b), we allow a flattening of the bright-end slope with redshift: $\alpha(z)=\alpha(0)+A z$. The resulting best-fit parameters are listed in the first line of Table 2 and the resulting model fit is presented in Fig. 9. The bright-end slope $\alpha$ derived by our fit ( $\alpha_{\mathrm{VVDS}}=-3.19$ at $\left.z=2.45\right)$ is consistent with that found by Richards et al. (2006b) $\left(\alpha_{\text {SDSS }}=-3.1\right)^{2}$.

\footnotetext{
${ }^{2}$ In their parameterization $A_{1}=-0.4(\alpha+1)=0.84$.
} 
Table 1. Binned $\log \Phi(B)$ luminosity-function estimates in the five redshift ranges. $\Delta M$ is the luminosity interval, $N_{\mathrm{AGN}}$ is the number of AGN in the bin and $\Delta \log \Phi(B)$ gives the $\pm 1 \sigma$ interval.

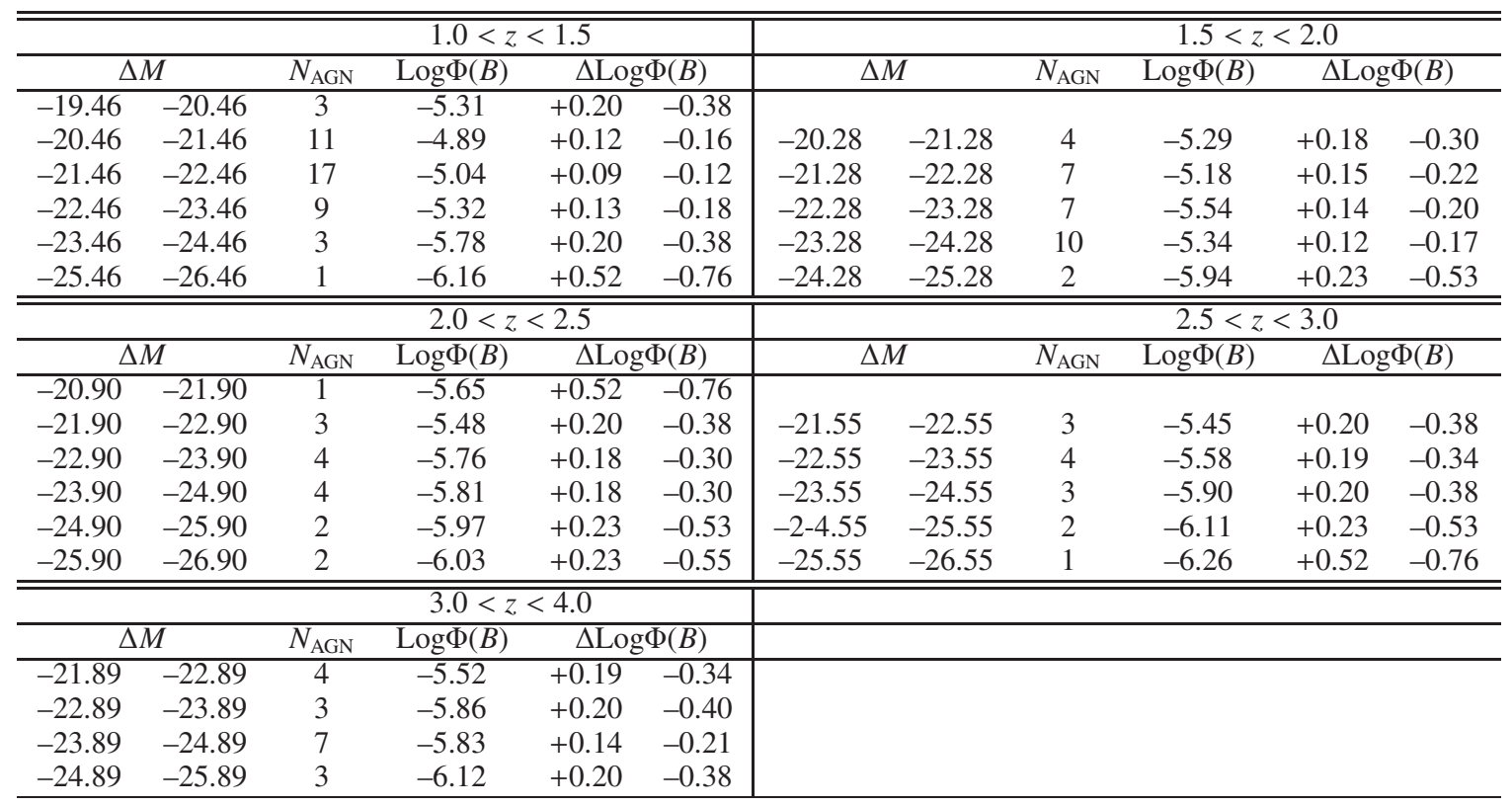

Table 2. Best fit models derived from the $\chi^{2}$ analysis of the combined sample VVDS+SDSS-DR3 in the redshift range $0.0<z<4.0$.

\begin{tabular}{cccccccccccc}
\hline \hline Sample - Evolution model & $\alpha$ & $\beta$ & $M^{*}$ & $k_{1 L}$ & $k_{2 L}$ & $A$ & $k_{1 \mathrm{D}}$ & $k_{2 \mathrm{D}}$ & $\Phi^{*}$ & $\chi^{2}$ & $v$ \\
\hline VVDS+SDSS - PLE $\alpha$ var & -3.83 & -1.38 & -22.51 & 1.23 & -0.26 & 0.26 & - & - & $9.78 \mathrm{E}-7$ & 130.36 & 69 \\
VVDS+SDSS - PLE+PDE & -3.49 & -1.40 & -23.40 & 0.68 & -0.073 & - & -0.97 & -0.31 & $2.15 \mathrm{E}-7$ & 91.4 & 68 \\
\hline Sample - Evolution model & $\alpha$ & $\beta$ & $M^{*}$ & $p 1$ & $p 2$ & $\gamma$ & $z_{\mathrm{c}, 0}$ & $M_{\mathrm{c}}$ & $\Phi^{*}$ & $\chi^{2}$ & $v$ \\
\hline VVDS+SDSS - LDDE & -3.29 & -2.0 & -24.38 & 6.54 & -1.37 & 0.21 & 2.08 & -27.36 & $2.79 \mathrm{E}-8$ & 64.6 & 67 \\
\hline
\end{tabular}

As shown in Fig. 9, this model reproduces well the bright part of the LF in the entire redshift range, but it does not fit the faint part at low redshift $(1.0<z<1.5)$. This appears to be due to the fact that the derived faint-end slope $(\beta=-1.38)$ is too shallow to reproduce the VVDS data.

Richards et al. (2005), working on a combined 2dF-SDSS (2SLAQ) sample of AGN up to $z=2.1$, found, fixing all of the parameters except $\beta$ and the normalization to those of Croom et al. (2004), a faint-end slope $\beta=-1.45 \pm 0.03$. This value would describe better our faint LF at low redshift. This trend suggests a kind of combined luminosity and density evolution not taken into account by this model. For this reason, we attempted to fit the data by adding a term of density evolution in the form of:

$\Phi_{M}^{*}(z)=\Phi_{M}^{*}(0) \cdot 10^{k_{1 \mathrm{D}} z+k_{2 \mathrm{D}} z^{2}}$.

The derived best fit parameters of this model are listed in the second line of Table 2 and the model fit is shown in Fig. 9. This model gives a better $\chi^{2}$ compared to the previous model, describing the entire sample better than a simple PLE (the reduced $\chi^{2}$ decreases from $\sim 1.9$ to $\sim 1.35$ ). However, it still does not satisfactorily reproduce the excess of faint objects in the redshift bin $1.0<z<1.5$ and it underestimates the faint end of the LF in the last redshift bin $(3.0<z<4.0)$.

\subsection{The LDDE model}

Recently, a growing number of observations at different redshifts, in soft and hard X-ray bands, have found evidences of a flattening of the faint-end slope of the LF towards high redshift. This trend has been described through a luminosity-dependent density evolution (LDDE) parameterization (Miyaji et al. 2000; Ueda et al. 2003; Hasinger et al. 2005). Such a parameterization allows the redshift of the AGN density peak to change as a function of luminosity. This could explain the excess of faint AGN found in the VVDS sample at $1.0<z<1.5$. Following Hasinger et al. (2005), we assume an LDDE evolution of the form:

$\Phi\left(M_{B}, z\right)=\Phi(M, 0) * e_{\mathrm{d}}\left(z, M_{B}\right)$

where:

$e_{\mathrm{d}}\left(z, M_{B}\right)= \begin{cases}(1+z)^{p 1} & \left(z \leq z_{\mathrm{c}}\right) \\ e_{\mathrm{d}}\left(z_{\mathrm{c}}\right)\left[(1+z) /\left(1+z_{\mathrm{c}}\right)\right]^{p 2} & \left(z>z_{\mathrm{c}}\right) .\end{cases}$

along with

$z_{\mathrm{c}}\left(M_{B}\right)= \begin{cases}z_{\mathrm{c}, 0} 10^{-0.4 \gamma\left(M_{B}-M_{\mathrm{c}}\right)} & \left(M_{B} \geq M_{\mathrm{c}}\right) \\ z_{\mathrm{c}, 0} & \left(M_{B}<M_{\mathrm{c}}\right) .\end{cases}$

where $z_{\mathrm{c}}$ corresponds to the redshift at which the evolution changes. Note that $z_{\mathrm{c}}$ is not constant, but depends on luminosity. This dependence allows different evolutions at different luminosities and can indeed reproduce the differential AGN evolution as a function of luminosity, thus modifying the shape of the luminosity function as a function of redshift. We also considered two different assumptions for $p 1$ and $p 2$ : (i) both parameters constant and (ii) both linearly depending on luminosity as follows:

$p 1\left(M_{B}\right)=p 1_{M_{\text {ref }}}-0.4 \epsilon_{1}\left(M_{B}-M_{\text {ref }}\right)$

$p 2\left(M_{B}\right)=p 2_{M_{\mathrm{ref}}}-0.4 \epsilon_{2}\left(M_{B}-M_{\mathrm{ref}}\right)$. 


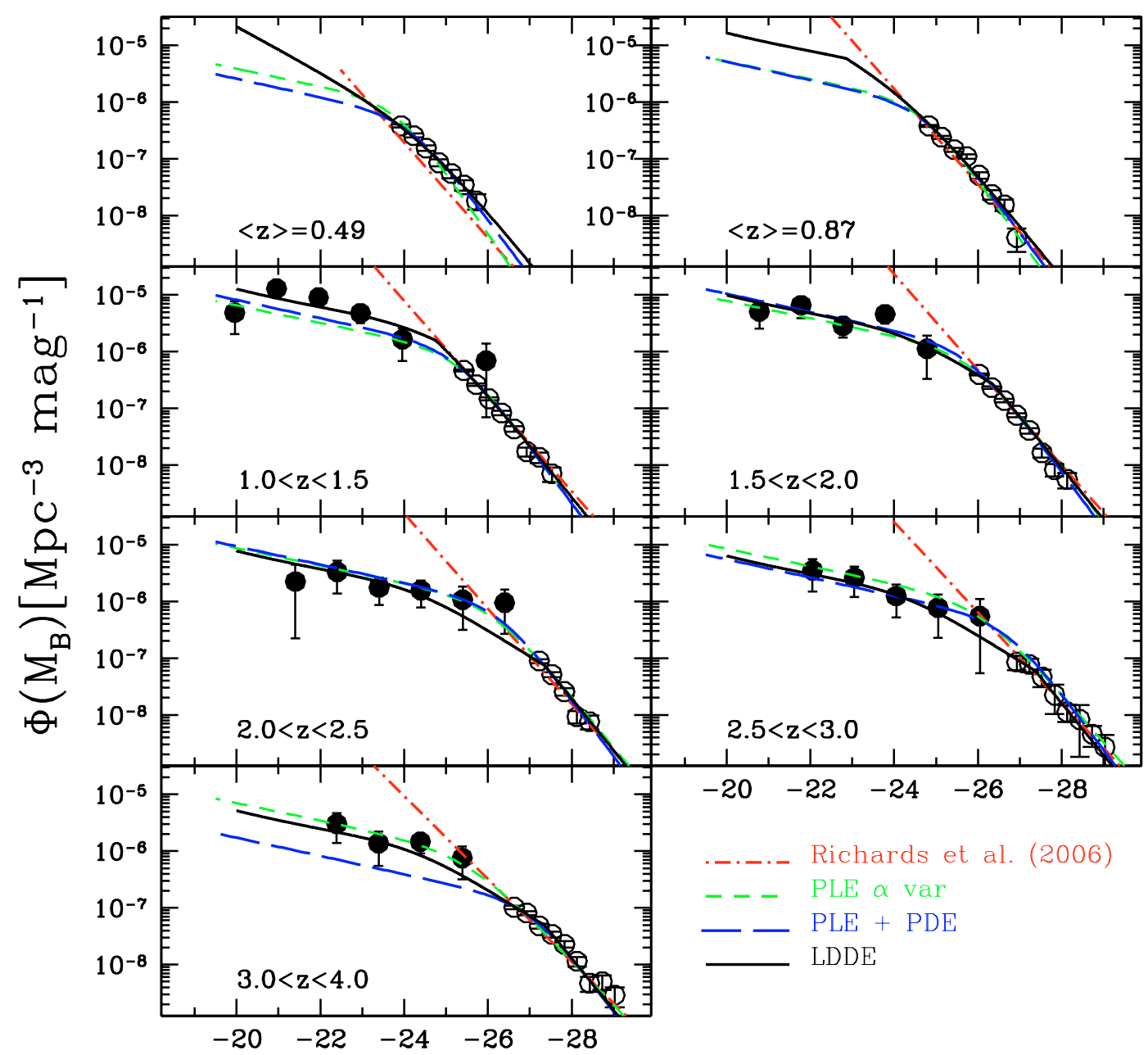

\section{Absolute Magnitude $\left(\mathrm{M}_{\mathrm{B}}\right)$}

Fig. 9. $B$-band luminiosity functions as a function of redshift from the VVDS (filled circles) and SDSS Data Release 3 (open circles Richards et al. 2006b). The red dot-dashed line corresponds to the model fit derived by Richards et al. (2006b) using the SDSS data only. The other lines correspond to model fits derived considering the combination of the VVDS and SDSS samples for different evolutionary models, as listed in Table 2 and described in Sect. 7.

The corresponding $\chi^{2}$ values for the above two cases are respectively $\chi^{2}=64.6$ and $\chi^{2}=56.8$. Given the relatively small improvement of the fit, we considered the addition of the two further parameters $\epsilon_{1}$ and $\epsilon_{2}$ unnecessary. The model with constant $p 1$ and $p 2$ values is shown Fig. 9 and the best fit parameters derived for this model are reported in the last line of Table 2.

This model reproduces well the overall shape of the luminosity function over the entire redshift range, including the excess of faint $\mathrm{AGN}$ at $1.0<z<1.5$. The $\chi^{2}$ value for the LDDE model is in fact the best among all the analyzed models. The fit is very good, with a $\chi^{2}$ of 64.6 for 67 degrees of freedom ${ }^{3}$.

The best fit value of the faint-end slope at $z=0$ is $\beta=-2.0$. This value is consistent with that derived by Hao et al. (2005) studying the emission-line luminosity function of a sample of Seyfert galaxies at very low redshift $(0<z<0.15)$ extracted from the SDSS. They derived slopes $\beta$ ranging from -2.07 to -2.03 , depending on the line ( $\mathrm{H} \alpha$, [O II] or [O III]) used to compute the nuclear luminosity. Moreover, the normalizations

3 We note that the reduced $\chi^{2}$ of our best fit model, which includes also VVDS data, is significantly better than that obtained by Richards et al. (2006b) in fitting only the SDSS DR3 data. are in good agreement, even though we have to rely on an extrapolation of the fitted model towards low redshifts not covered by the VVDS.

\section{AGN activity as a function of redshift}

By integrating the luminosity function corresponding to our best fit model (i.e. the LDDE model; see Table 2), we derive the comoving AGN space density as a function of redshift for different luminosity ranges Fig. 10).

The existence of a peak at $z \sim 2$ in the space density of bright AGN is known since a long time, even if it is difficult to locate precisely its position within a single optical survey. Figure 10 shows that for our best-fit model the AGN peak space density shifts significantly towards lower luminosity at low redshift. The position of the maximum moves from $z \sim 2.0$ for $M_{B}<-26.0$ to $z \sim 0.65$ for $-22<M_{B}<-20$.

A similar trend has recently been found in several deep X-ray selected samples (Cowie et al. 2003; Hasinger et al. 2005; La Franca et al. 2005). To compare with X-ray results we derive the space densities from our best fit LDDE model in the same bolometric-luminosity ranges as in La Franca et al. (2005). We 


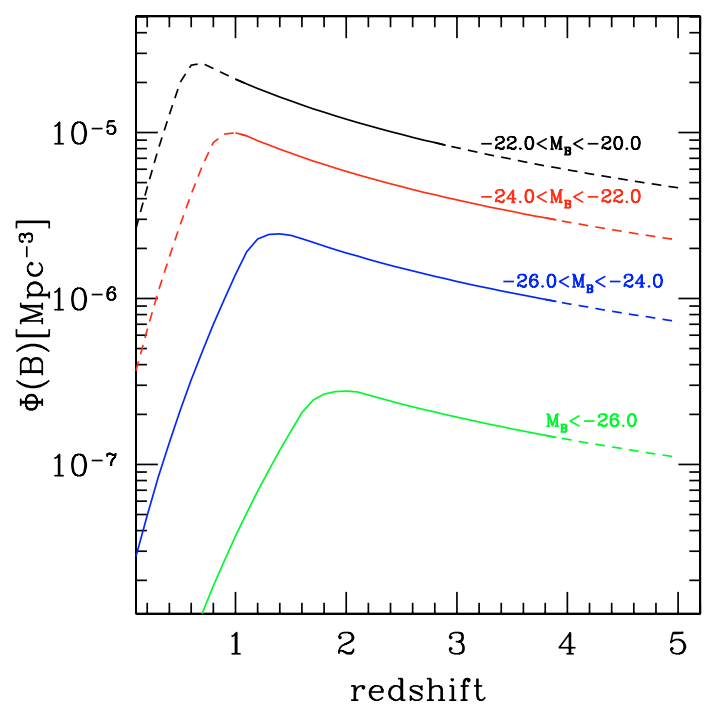

Fig. 10. Evolution of comoving AGN space density with redshift, for different luminosity range: $-22.0<M_{B}<-20.0 ;-24.0<M_{B}<$ $-22.0 ;-26.0<M_{B}<-24.0$ and $M_{\mathrm{B}}<-26.0$. Dashed lines correspond to the redshift range in which the model has been extrapolated.

found that the space density peaks at $z \simeq[0.35 ; 0.7 ; 1.1 ; 1.5]$ respectively for $\log L_{X(2-10 \mathrm{kev})}=[42-43 ; 43-44 ; 44-44.5$; 44.5-45]. In the same luminosity intervals, the values for the redshift of the peak obtained by La Franca et al. (2005) are $z \simeq[0.5 ; 0.8 ; 1.1 ; 1.5]$, in good agreement with our result. This trend has been interpreted as evidence of AGN (i.e. black hole) "cosmic downsizing", similar to what has recently been observed in the galaxy spheroid population (Cimatti et al. 2006).

As already said, this effect had not been seen so far in the analysis of optically selected samples. This can be due to the fact that most optical samples are limited to high-luminosity objects. The VVDS sample, being about one magnitude fainter than the COMBO-17 sample and not having any bias in finding faint AGN, allows us to detect for the first time in an optically selected sample the shift of the maximum space density towards lower redshift for low-luminosity AGN.

\section{Summary and conclusion}

In the present paper we have used the new sample of AGN collected by the VVDS and presented in Gavignaud et al. (2006) to derive the optical luminosity function of faint type-1 AGN.

The sample consists of 130 broad-line AGN selected solely on the basis of their spectral features, without morphological and/or color selection biases. The absence of these biases is particularly important, because the continuum emission of lowluminosity AGN can be masked by host-galaxy emission, making the optical selection of faint AGN candidates very difficult. Only spectroscopic surveys without any pre-selection can therefore be considered complete in this luminosity range.

Our sample shows redder colors than those expected, for example, on the basis of the color track derived from the SDSS composite spectrum, and the difference is stronger for the intrinsically faint objects. Thanks to the extended multi-wavelength coverage in the deep VVDS fields we examined the spectral energy distribution of each object and we fitted it with a combination of AGN and galaxy emission, allowing for a possible extinction of the AGN flux. We found that both effects (presence of dust and contamination from the host galaxy) are likely to be responsible for this reddening, even if it is not possible to exclude the possibility that faint AGN are intrinsically redder than brighter ones.

We derived the luminosity function in the $B$-band for $1<$ $z<3.6$, using the usual $1 / V_{\max }$ estimator (Schmidt 1968), which gives the space density contributions of individual objects. Moreover, using the prescriptions recently derived by Hopkins et al. (2007), we computed the bolometric luminosity function for our sample. This allowed us to compare our results with other samples selected from different bands.

Our sample, which is more than one magnitude fainter than previous optical surveys, allows us to constrain the faint part of the luminosity function up to high redshift. A comparison of our data with the $2 \mathrm{dF}$ sample at low redshift $(1<z<2.1)$ shows that the VVDS data cannot be fitted with the PLE models derived from previous samples. Qualitatively, our data suggest the presence of an excess of faint objects at low redshift $(1.0<z<1.5)$ with respect to these models.

Recently, a growing number of observations in soft and hard $\mathrm{X}$-ray bands have found evidence of a similar trend, which has been parameterized with a luminosity-dependent density evolution. This allows the redshift of the AGN density peak to change with luminosity and explains the excess of faint AGN that we found at $1.0<z<1.5$. Indeed, by combining our faint VVDS sample with the large sample of bright AGN extracted from the SDSS DR3 (Richards et al. 2006b), we found that the evolutionary model which better represents the combined luminosity functions, over a wide range of redshift and luminosity, is an LDDE model, similar to those derived from the major $\mathrm{X}$-surveys. The derived faint-end slope at $z=0$ is $\beta=-2.0$, consistent with the value derived by Hao et al. (2005) for the emission-line luminosity function of Seyfert galaxies at very low redshift.

A feature intrinsic to these LDDE models is that the peak of the comoving AGN space density moves with luminosity, in the sense that more luminous AGN peak earlier in the history of the Universe (i.e. at higher redshift). In particular, in our bestfit LDDE model the space-density peak ranges from $z \sim 2$ for $M_{B}<-26$ to $z \sim 0.65$ for $-22<M_{B}<-20$. This effect had not been seen so far in the analysis of optically selected samples because most of the optical samples do not sample adequately the faintest luminosities, which are needed to accurately locate the density peak.

Although the results presented here appear to be robust, the larger AGN sample we will have at the end of the still on-going VVDS survey (>300 AGN), will allow a better statistical analysis and a better estimate of the model parameters.

Acknowledgements. This research has been developed within the framework of the VVDS consortium.

This work has been partially supported by the CNRS-INSU and its Programme National de Cosmologie (France), and by Italian Ministry (MIUR) grants COFIN2000 (MM02037133) and COFIN2003 (num. 2003020150).

Based on data obtained with the European Southern Observatory Very Large Telescope, Paranal, Chile, program 070.A-9007(A), 272.A-5047, 076.A-0808, and on data obtained with MegaPrime/MegaCam, a joint project of CFHT and CEA/DAPNIA, at the Canada-France-Hawaii Telescope (CFHT) which is operated by the National Research Council (NRC) of Canada, the Institut National des Science de l'Univers of the Centre National de la Recherche Scientifique (CNRS) of France, and the University of Hawaii. This work is based in part on data products produced at TERAPIX and the Canadian Astronomy Data Centre as part of the Canada-France-Hawaii Telescope Legacy Survey, a collaborative project of NRC and CNRS.

The VLT-VIMOS observations have been carried out on guaranteed time (GTO) allocated by the European Southern Observatory (ESO) to the VIRMOS consortium, under a contractual agreement between the Centre National de la Recherche Scientifique of France, heading a consortium of French and Italian institutes, and ESO, to design, manufacture and test the VIMOS instrument. 


\section{References}

Arnouts, S., Schiminovich, D., Ilbert, O., et al. 2005, ApJ, 619, L43 Avni, Y., \& Bahcall, J. N. 1980, ApJ, 235, 694A

Barger, A. J., Cowie, L. L., Mushotzky, R. F., et al. 2005, AJ, 129, 578 Boyle, B. J., Shanks, T., \& Peterson, B. A. 1988, MNRAS, 235, 935 Boyle, B. J., Shanks, T., Croom, S. M., et al. 2000, MNRAS, 317, 1014 Brandt, W. N., Hornschemeier, A. E., Schneider, D. P., et al. 2000, AJ, 119, 2349 Brown, M. J. I., Brand, K., Dey, A., et al. 2006, ApJ, 638, 88 Bruzual, G., \& Charlot, S. 2003, MNRAS, 344, 1000

Cimatti, A., Daddi, E., \& Renzini, A. 2006, A\&A, 453, L29 Cirasuolo, M., Magliocchetti, M., \& Celotti, A. 2005, MNRAS, 357, 1267

Cowie, L. L., Barger, A. J., Bautz, M. W., Brandt, W. N., \& Garmire, G. P. 2003, ApJ, 584, L57

Croom, S. M., Smith, R. J., Boyle, B. J., et al. 2004, MNRAS, 349, 1397

Di Matteo, T., Springel, V., \& Hernquist, L. 2005, Nature, 433, 604

Fan, X., Strauss, M. A., Schneider, D. P., et al. 2001, AJ, 121, 54

Ferrarese, L., \& Merritt, D. 2000, ApJ, 539, L9

Fiore, F., Brusa, M., Cocchia, F., et al. 2003, A\&A, 409, 79

Fontanot, F., Cristiani, S., Monaco, P., et al. 2007, A\&A, 461, 39

Gavignaud, I., Bongiorno, A., Paltani, S., et al. 2006, A\&A, 457, 79

Hall, P. B., Gallagher, S. C., Richards, G. T., et al. 2006, AJ, 132, 1977

Hao, L., Strauss, M. A., Fan, X., et al. 2005, AJ, 129, 1795

Hartwick, F. D. A., \& Schade, D. 1990, ARA\&A, 28, 437

Hasinger, G., Miyaji, T., \& Schmidt, M. 2005, A\&A, 441, 417

Hewett, P. C., \& Foltz, C. B. 2003, AJ, 125, 1784

Hewett, P. C., Foltz, C. B., Chaffee, F. H., et al. 1991, AJ, 101, 1121

Hopkins, P. F., Hernquist, L., Cox, T. J., et al. 2006, ApJ, 639, 700

Hopkins, P. F., Richards, G. T., \& Hernquist, L. 2007, ApJ, 654, 731

Hunt, M. P., Steidel, C. C., Adelberger, K. L., \& Shapley, A. E. 2004, ApJ, 605, 625

Ilbert, O., Tresse, L., Zucca, E., et al. 2005, A\&A, 439, 863

Iovino, A., McCracken, H. J., Garilli, B., et al. 2005, A\&A, 442, 423

Kennefick, J. D., Djorgovski, S. G., \& de Carvalho, R. R. 1995, AJ, 110, 2553

Kormendy, J., \& Richstone, D. 1995, ARA\&A, 33, 581

La Franca, F., Fiore, F., Comastri, A., et al. 2005, ApJ, 635, 864

La Franca, F., Fiore, F., Vignali, C., et al. 2002, ApJ, 570, 100

Lawrence, A., Warren, S. J., Almaini, O., et al. 2006, ArXiv Astrophysics e-prints

Le Fèvre, O., Mellier, Y., McCracken, H. J., et al. 2004a, A\&A, 417, 839

Le Fèvre, O., Vettolani, G., Paltani, S., et al. 2004b, A\&A, 428, 1043

Le Fèvre, O., Vettolani, G., Garilli, B., et al. 2005, A\&A, 439, 845

Lonsdale, C. J., Smith, H. E., Rowan-Robinson, M., et al. 2003, PASP, 115, 897

Lonsdale, C., Polletta, M. d. C., Surace, J., et al. 2004, ApJS, 154, 54

Magorrian, J., Tremaine, S., Richstone, D., et al. 1998, AJ, 115, 2285

Marshall, H. L., Tananbaum, H., Avni, Y., \& Zamorani, G. 1983, ApJ, 269, 35

Matute, I., La Franca, F., Pozzi, F., et al. 2006, A\&A, 451, 443

McCracken, H. J., Radovich, M., Bertin, E., et al. 2003, A\&A, 410, 17

Miyaji, T., Hasinger, G., \& Schmidt, M. 2000, A\&A, 353, 25

Miyaji, T., Hasinger, G., \& Schmidt, M. 2001, A\&A, 369, 49

Mushotzky, R. F., Cowie, L. L., Barger, A. J., \& Arnaud, K. A. 2000, Nature, 404,459

Osterbrock, D. E. 1981, ApJ, 249, 462

Page, M. J., Mason, K. O., McHardy, I. M., Jones, L. R., \& Carrera, F. J. 1997, MNRAS, 291, 324

Pei, Y. C. 1995, ApJ, 438, 623

Prevot, M. L., Lequeux, J., Prevot, L., Maurice, E., \& Rocca-Volmerange, B. 1984, A\&A, 132, 389

Radovich, M., Arnaboldi, M., Ripepi, V., et al. 2004, A\&A, 417, 51

Richards, G. T., Fan, X., Newberg, H. J., et al. 2002, AJ, 123, 2945

Richards, G. T., Hall, P. B., Vanden Berk, D. E., et al. 2003, AJ, 126, 1131

Richards, G. T., Croom, S. M., Anderson, S. F., et al. 2005, MNRAS, 360, 839

Richards, G. T., Lacy, M., Storrie-Lombardi, L. J., et al. 2006a, ApJS, 166, 470

Richards, G. T., Strauss, M. A., Fan, X., et al. 2006b, AJ, 131, 2766

Scannapieco, E., \& Oh, S. P. 2004, ApJ, 608, 62

Schiminovich, D., Ilbert, O., Arnouts, S., et al. 2005, ApJ, 619, L47

Schlegel, D. J., Finkbeiner, D. P., \& Davis, M. 1998, ApJ, 500, 525

\section{Schmidt, M. 1968, ApJ, 151, 393}

Schmidt, M., Schneider, D. P., \& Gunn, J. E. 1995, AJ, 110, 68 Schneider, D. P., Fan, X., Hall, P. B., et al. 2003, AJ, 126, 2579

Siana, B., Polletta, M., Smith, H. E., et al. 2006, ArXiv Astrophysics e-prints Silk, J., \& Rees, M. J. 1998, A\&A, 331, L1

Silverman, J. D., Green, P. J., Barkhouse, W. A., et al. 2005, ApJ, 624, 630

Steidel, C. C., Hunt, M. P., Shapley, A. E., et al. 2002, ApJ, 576, 653

Ueda, Y., Akiyama, M., Ohta, K., \& Miyaji, T. 2003, ApJ, 598, 886

Vanden Berk, D. E., Richards, G. T., Bauer, A., et al. 2001, AJ, 122, 549

Warren, S. J., Hewett, P. C., \& Osmer, P. S. 1994, ApJ, 421, 412

Wolf, C., Wisotzki, L., Borch, A., et al. 2003, A\&A, 408, 499

1 Università di Bologna, Dipartimento di Astronomia, via Ranzani 1, 40127, Bologna, Italy

e-mail: angela.bongiorno@oabo.inaf.it

2 INAF - Osservatorio Astronomico di Bologna, via Ranzani 1, 40127 Bologna, Italy

3 Astrophysical Institute Potsdam, An der Sternwarte 16, 14482 Potsdam, Germany

${ }^{4}$ Integral Science Data Centre, Ch. d'Écogia 16, 1290 Versoix, Switzerland

5 Geneva Observatory, University of Geneva, Ch. des Maillettes 51, 1290 Sauverny, Switzerland

${ }^{6}$ Laboratoire d'Astrophysique de Toulouse/Tabres (UMR5572), CNRS, Université Paul Sabatier - Toulouse III, Observatoire MidiPyrénées, 14 Av. E. Belin, 31400 Toulouse, France

7 Institute for Astronomy, University of Edinburgh, Royal Observatory, Edinburgh EH9 3HJ, UK

${ }^{8}$ IASF-INAF, via Bassini 15, 20133 Milano, Italy

9 Laboratoire d'Astrophysique de Marseille, UMR 6110 CNRS Université de Provence, BP 8, 13376 Marseille Cedex 12, France

10 IRA-INAF, via Gobetti 101, 40129 Bologna, Italy

11 INAF - Osservatorio Astronomico di Roma, via di Frascati 33, 00040 Monte Porzio Catone, Italy

12 Max Planck Institut fur Astrophysik, 85741 Garching, Germany

13 Institut d'Astrophysique de Paris, UMR 7095, 98bis Bd. Arago, 75014 Paris, France

14 School of Physics \& Astronomy, University of Nottingham, University Park, Nottingham NG72RD, UK

15 INAF - Osservatorio Astronomico di Brera, via Brera 28, Milan, Italy

${ }^{16}$ Institute for Astronomy, 2680 Woodlawn Dr., University of Hawaii, Honolulu 96822, Hawaii

17 Observatoire de Paris, LERMA, 61 avenue de l'Observatoire, 75014 Paris, France

18 Centre de Physique Théorique, UMR 6207 CNRS-Université de Provence, 13288 Marseille France

19 Astronomical Observatory of the Jagiellonian University, ul Orla 171, 30-244 Kraków, Poland

20 INAF - Osservatorio Astronomico di Capodimonte, via Moiariello 16, 80131 Napoli, Italy

21 European Southern Observatory, Karl-Schwarzschild-Strasse 2, 85748 Garching bei München, Germany

${ }^{22}$ Center for Astrophysics \& Space Sciences, University of California, San Diego, La Jolla, CA 92093-0424, USA

${ }^{23}$ Centro de Astrofísica da Universidade do Porto, Rua das Estrelas, 4150-762 Porto, Portugal

${ }^{24}$ Universitá di Milano-Bicocca, Dipartimento di Fisica - Piazza delle Scienze 3, 20126 Milano, Italy

25 Università di Bologna, Dipartimento di Fisica, via Irnerio 46, 40126 Bologna, Italy 